\title{
Is Gravitational Radiation a Radiation of the Same Level as Electromagnetic Radiation?
}

\author{
Stanislav I. Fisenko, I. S. Fisenko \\ Rusthermosynthesis JSC, Moscow, Russia \\ Email: StanislavFisenko@yandex.ru
}

Received 28 May 2014; revised 27 June 2014; accepted 21 July 2014

Copyright (C) 2014 by authors and Scientific Research Publishing Inc.

This work is licensed under the Creative Commons Attribution International License (CC BY). http://creativecommons.org/licenses/by/4.0/

(c) (7) Open Access

\begin{abstract}
The notion of gravitational radiation as a radiation of the same level as the electromagnetic radiation is based on the theoretically proven and experimentally confirmed facts of the existence of stationary states of an electron in its gravitational field, characterized by the gravitational constant $K=1^{42} G$ ( $G$ is Newtonian gravitational constant) and unrecoverable space-time curvature $\Lambda$. These experimental facts include, in particular, data on the broadening of the spectra of the characteristic radiation of multielectron atoms. This broadening of the spectra can be only due to the additional broadening mechanism, in particular the presence of excited states of electrons in their gravitational field. Another fact is the new line in the X-ray emission spectrum according to the results of observation with MOS-camera of the XMM-Newton observatory. This line unlike other identified lines of electromagnetic radiation cannot be assigned to any atomic transition.
\end{abstract}

\section{Keywords}

Electron, Stationary State, Characteristic Radiation, The Broadening of the Emission Spectra

\section{Introduction}

Numerous attempts to detect experimentally gravitational radiation (as based on the notions of the general relativity theory and alternative theories) brought no result. Studying the development of the relativistic theory of gravitation (which is the relativistic theory of gravitation, and not any of its particular case, such as general relativity theory), the authors obtained a model of gravitational interaction at the quantum level, which has no analogues [1] [2]. This model detailed in these papers made it possible to calculate the spectrum of gravitational radiation and its subsequent interpretation based on the available experimental data. 


\section{The Energy Spectrum of Stationary States of an Electron in Its Gravitational Field}

The energy spectrum of an electron in its gravitational field calculated in the simplest approximation [1] is shown in Figure 1. Figure 2 shows the geometrical interpretation of the model of an electron in its gravitational field. The obtained results indicate gravitational radiation as a radiation of one level with electromagnetic radiation as follows from the nature of the electron energy spectrum in its gravitational field shown in Figure 1. Further, the following circumstances are in particular important.

a) The presence of cascade transitions from the top down leading to a partial transfer of the gravitational radiation spectrum to longer wavelengths.

b) The broadening of the spectra of characteristic electromagnetic radiation due to the increased likelihood of ion transitions to other states.

c) The energy spectrum of the electron in its own gravitational field (Figure 1) and the energy spectra of multielectron atoms (Figure 3) are such that there is a resonance of these spectra. As obvious, the consequence of such resonant interaction is appearance, including new lines, of electromagnetic transitions not associated with atomic transitions.

\section{Broadening Spectra of Characteristic Radiation}

Broadening of spectral lines of micropinches arising in plasma of multicharge ions [3] [4] is not consistent with existing electromagnetic concepts. As the mechanisms of spectral line broadening, in [3] [4] Doppler, radiative and collisional broadening were considered. Such a fit to these mechanisms of the broadening does not lead to a complete reproduction of the registered part of the emission spectrum of a micropinch. This is an evidence of the existence of an additional mechanism of broadening due to excited states of electrons. The corresponding por-

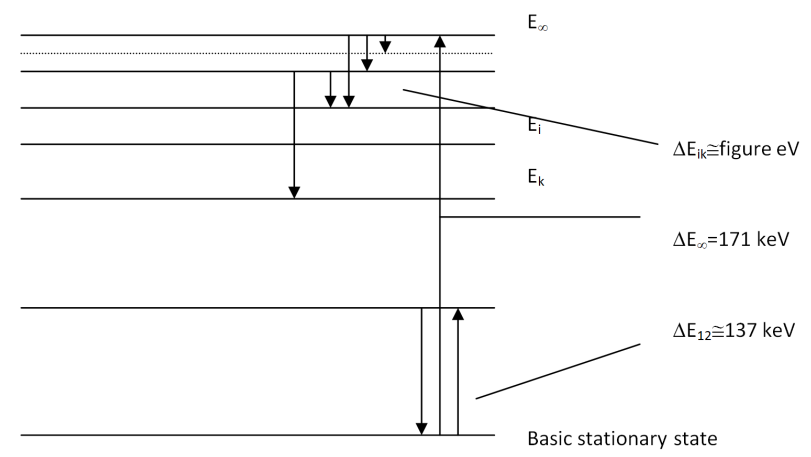

Figure 1. Transitions to stationary states of electrons in proper gravitational field.

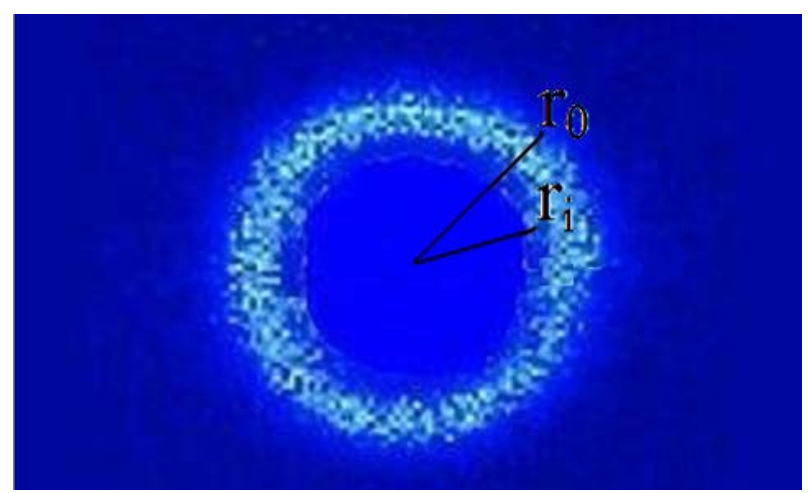

Figure 2. Electron in proper gravitational field in the ground stationary state. $\mathrm{r}_{\mathrm{i}}$-radius of irremovable curvature, $\mathrm{r}_{0}$ - "classical" electron radius being a radius of the ground stationary state of electron in proper gravitational field. 
tion of the spectrum of gravitational radiation already has no clearly pronounced lines due to the transfer of energy in the spectrum in the long wavelength region.

In other words, said additional broadening mechanism of spectral lines of the characteristic electromagnetic radiation of multicharge ions (in conditions of compressing plasma by emitted gravitational field) is the sole and unquestionable method of quenching excited states of electrons with energy levels of ion emission, and excitation of these levels by gravitational radiation at resonant frequencies. Such increase in the probability of transitions of the ions to other states leads to an additional broadening of the spectral lines of the characteristic radiation. The reason for the rapid degradation of micropinches in a variety of high-current pulsed discharges with multicharge ions is also clear. Here we have only partial thermalization of accelerated plasma, when the power of gravitational radiation is insufficient to support more steady states of plasma [2].

\section{New Lines in the Spectra of X-Ray Radiation}

Two groups of researchers, independently of each other [5] [6] reported that the in the X-ray spectra of clusters of galaxies, a new line of radiation with an energy of $3.57 \mathrm{keV}$ was discovered.

This radiation should go from hot intergalactic gas that fills the cluster of galaxies, but, unlike other identified emission lines, this one cannot be assigned [3] to any atomic transition. The results of these measurements are shown in Figure 4 and Figure 5. At the same time, the resonance spectra of stationary states of electrons in their

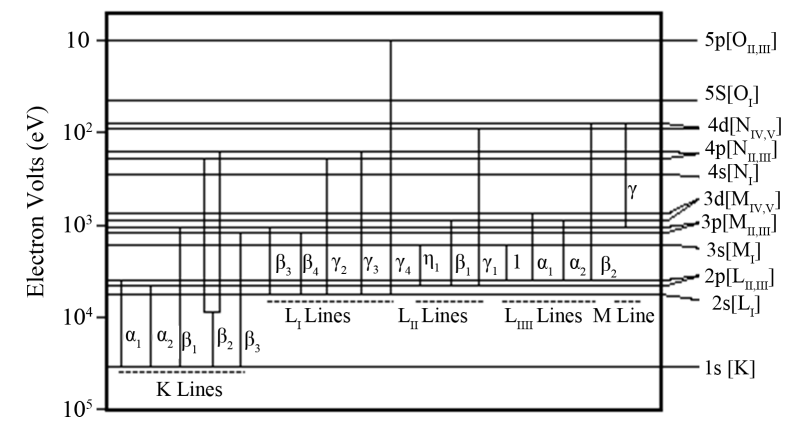

Figure 3. Scheme of K-, L- and M-levels of energy of the atom, and the main lines of K- and L-series. The energies of photons of the main lines reach units and scores of $\mathrm{keV}$.

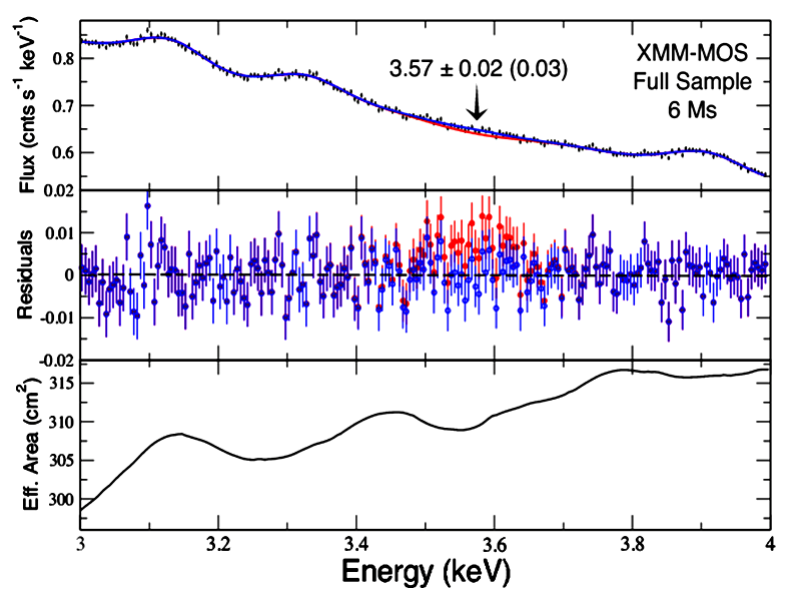

Figure 4. Above: MOS-camera spectrum in the range of 3 to $4 \mathrm{keV}$, XMM-Newton observatory .Separate dashes are the results of observation with errors; red curve is the best reproduction of the spectrum when only known emission lines of ions taken into account, blue curve is the result of addition of another previously unknown emission line. Bottom: deviation of observation data from the red and blue curves [5]. 
gravitational field and the spectra of multicharge ions [2] can provide not only the registered emission line, but also other lines of such properties. We can expect the presence of such lines in detailed registration of the emission spectra of astrophysical objects in the energy range greater than $8 \mathrm{keV}$.

Figure 6 shows the results of earlier measurements of the soft X-ray spectrum ([7], 1997). There is no new
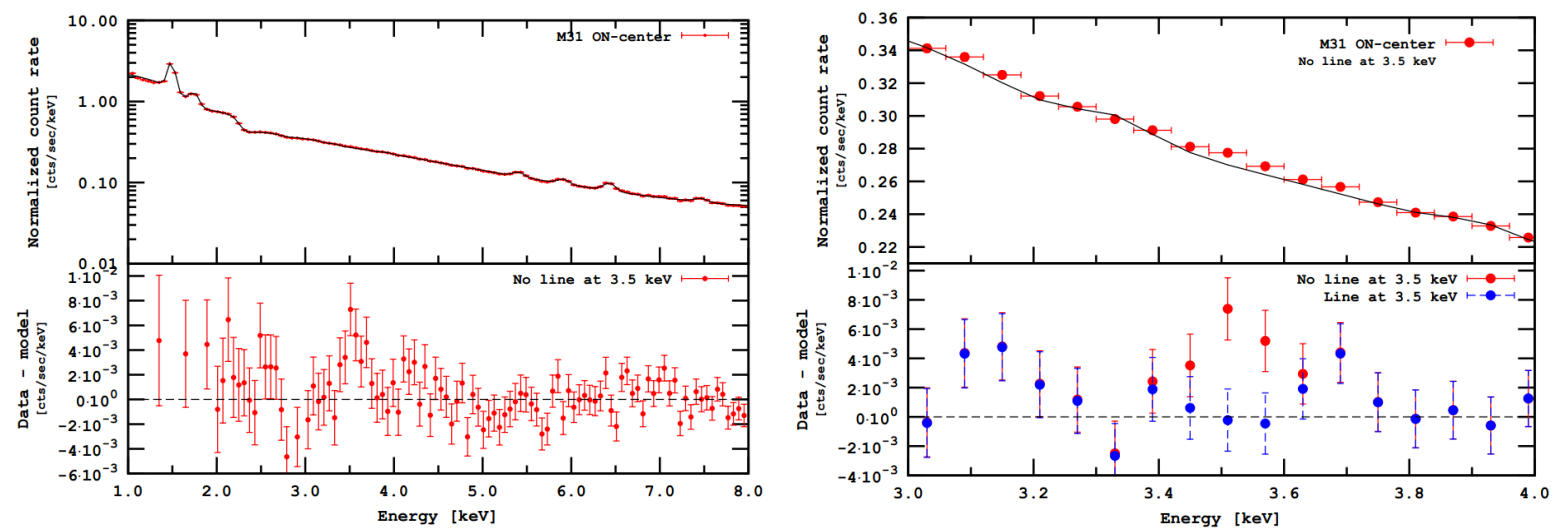

Figure 5. X-ray spectrum of the central part of the Andromeda nebula according to the results of observation with MOScamera of XMM-Newton observatory. Left: the entire spectrum from 1 to $8 \mathrm{keV}$, Right: the range of 3 to $4 \mathrm{keV}$. Symbols are the same as in Figure 4, [6].

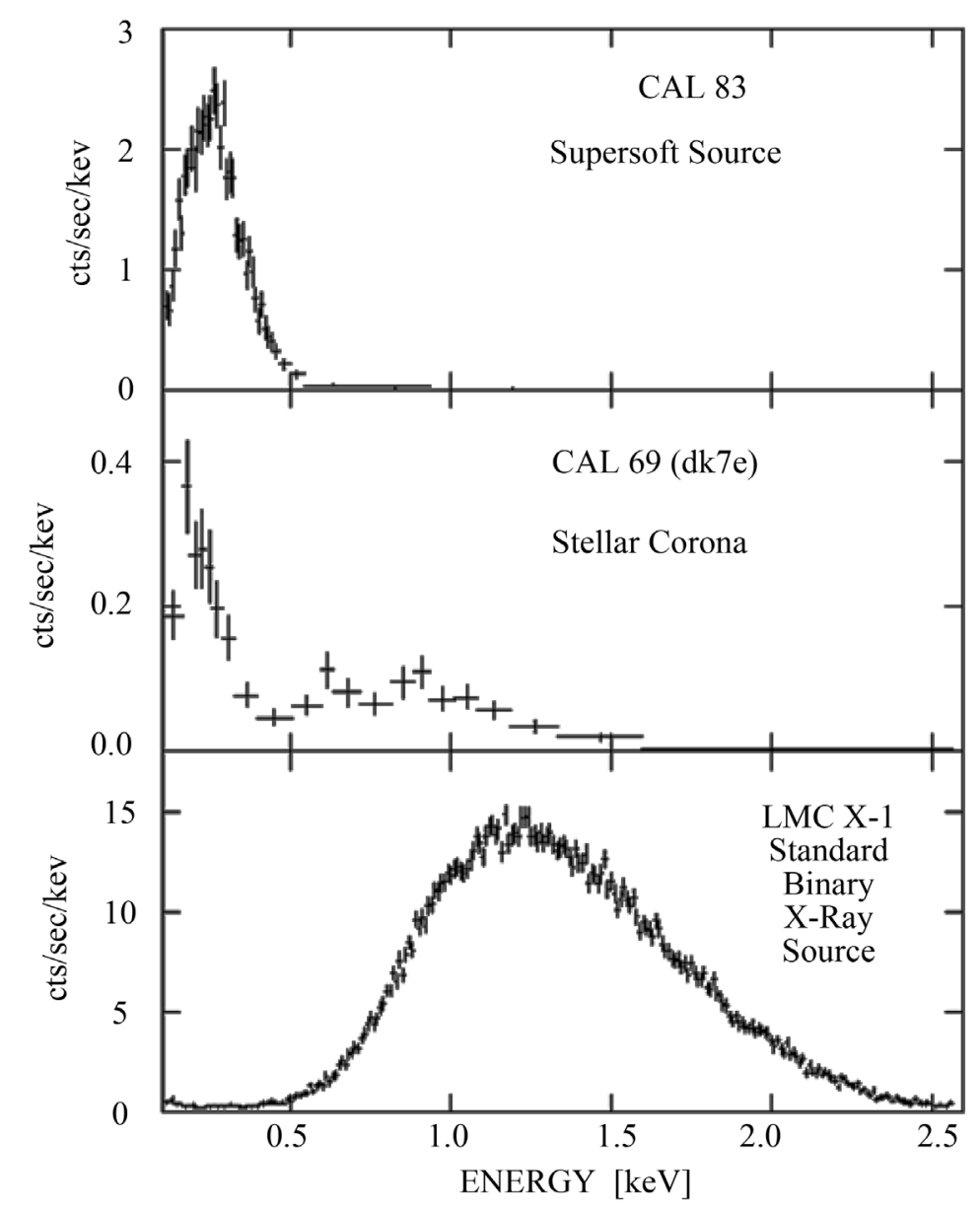

Figure 6. X-ray spectra of various X-ray sources: supersoft source, Stellar Corona, and the classical source [7]. 
line in this spectrum, but the spectrum is clearly broadened, more than it would be consistent with known mechanisms of spectral broadening [2].

\section{References}

[1] Fisenko, S.I. and Fisenko, I.S. (2012) International Journal of Theoretical and Applied Physics (IJTAP), 2, 32-39.

[2] Fisenko, S.I. and Fisenko, I.S. (2013) Journal of Modern Physics, 4, 481-485.

[3] Politov, V.Yu., Potapov, A.V. andAntonova, L.V. (1998) Proceeding of International Conference "V Zababakhin Scientific Proceedings”, 28-34.

[4] Haines, M.G., et al. (2006) Physical Review Letters, 96, 075003-075008.

[5] Bulbul, E., et al. (2014) Detection of An Unidentified Emission Line in the Stacked X-ray spectrum of Galaxy Clusters. arXiv:1402.2301 [astro-ph.CO]

[6] Boyarsky A., Ruchayskiy O., Iakubovskyi D. and Franse J. (2014) An Unidentified Line in X-ray Spectra of the Andromeda Galaxy and Perseus Galaxy Cluster. arXiv:1402.4119 [astro-ph.CO]

[7] Kahabka, P. and van den Heuvel, E.P.J. (1997) Annual Review of Astronomy and Astrophysics, 35, 69-100. http://dx.doi.org/10.1146/annurev.astro.35.1.69 
Scientific Research Publishing (SCIRP) is one of the largest Open Access journal publishers. It is currently publishing more than 200 open access, online, peer-reviewed journals covering a wide range of academic disciplines. SCIRP serves the worldwide academic communities and contributes to the progress and application of science with its publication.

Other selected journals from SCIRP are listed as below. Submit your manuscript to us via either submit@scirp.org or Online Submission Portal.
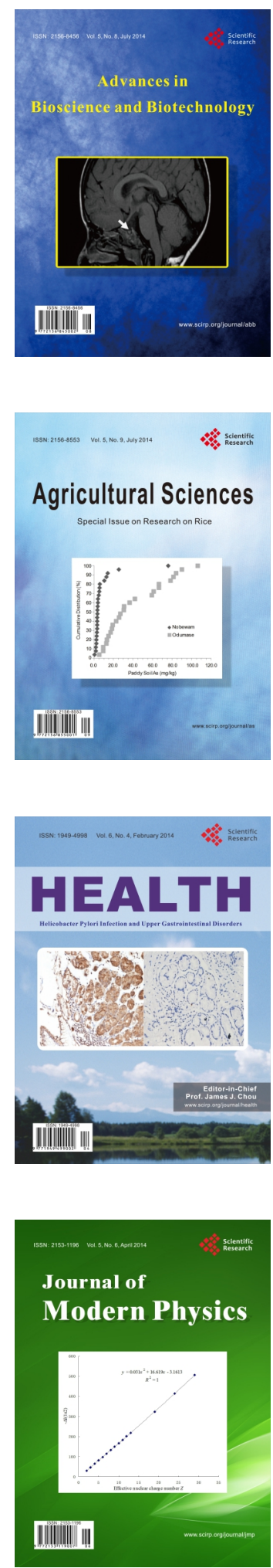
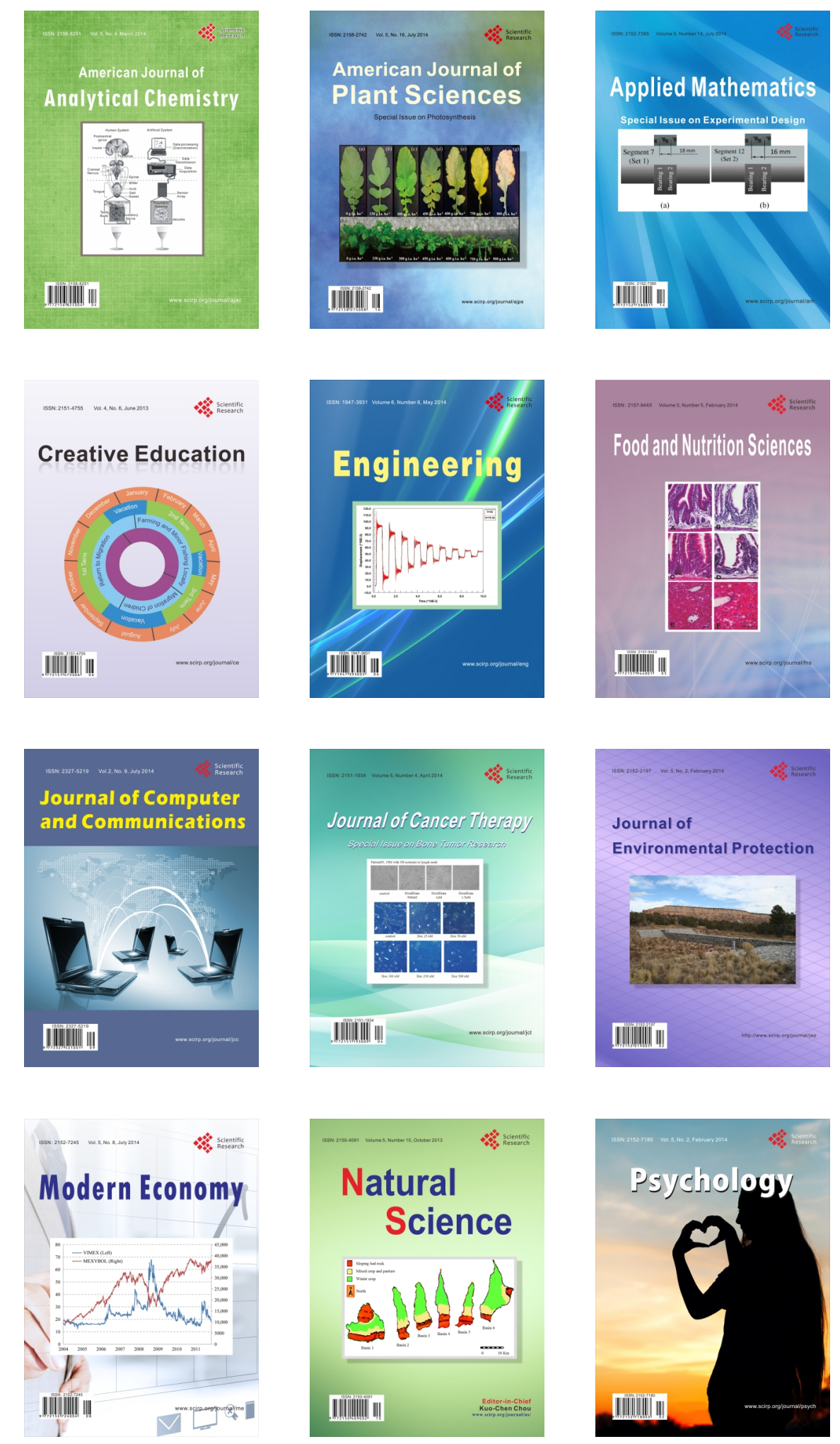